\title{
Towards Process-Aware Cross-Organizational Human Resource Management ${ }^{\star}$
}

\author{
Cristina Cabanillas ${ }^{1}$, Alex Norta ${ }^{2}$, Manuel Resinas ${ }^{3}$, \\ Jan Mendling ${ }^{1}$ and Antonio Ruiz-Cortés ${ }^{3}$ \\ ${ }^{1}$ Vienna University of Economics and Business, Austria \\ \{cristina.cabanillas, jan.mendling\}@wu.ac.at \\ ${ }^{2}$ Tallinn University of Technology, Estonia alex.norta@gmail.com \\ ${ }^{3}$ University of Seville, Spain \{resinas, aruiz\}@us.es
}

\begin{abstract}
Finding human resources with the required set of skills, experience, and availability to execute an activity at a specific moment, is a socio-technical challenge for enterprises that use business-process aware systems. On an intra-organizational level, there exists an increasing body of knowledge for automated human-resource management. However, the recent pervasiveness of service-oriented cloud computing combined with mobile devices and big data, has resulted in the emergence of crossorganizational ecosystems in which workforce is distributed. Consequently, human-resource management has to consider more requirements compared to a purely intra-organizational setting. This position paper addresses the gap and describes a set of challenges in the management of human resources in service outsourcing scenarios based on process views and automatic process-view matching. The contribution is a specification of research directions that must be pursued so that resource management successfully adopts the special requirements for scaling to a cross-organizational level.
\end{abstract}

Keywords: human resource management, process matching, process view, resource allocation, resource assignment, service outsourcing

\section{Introduction}

For running human resource management in Virtual Enterprises(VEs) [1], new enabling concepts and technologies are available in the form of Service-Oriented Cloud Computing (SOCC) [2]. This is facilitated via the use of Business Process as a Service (BPaaS) [3] that helps to choreograph service interactions in the VEs. Organizations that engage in a VE configuration for the purpose of carrying out business transactions have an interest in disclosing only what is necessary. Otherwise, details of Business Processes (BPs) on an in-house level must remain opaque for protecting business secrets that represent competitive advantages.

\footnotetext{
* This work was partially supported by the European Union's Seventh Framework Programme (FP7/2007-2013), the European Commission (FEDER), the Spanish and the Andalusian R\&D\&I programmes (grants 318275 (GET Service), P12-TIC1867 (COPAS), TIN2012-32273 (TAPAS), TIC-5906 (THEOS)).
} 


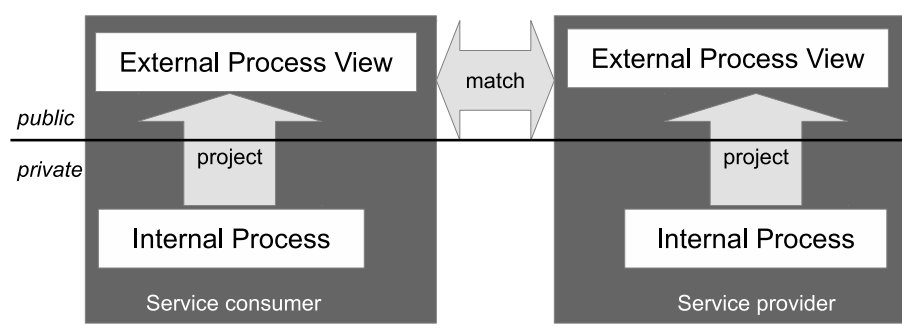

Fig. 1: A specification framework for service outsourcing [4].

A disclosure of the latter usually results in an organization loosing its base for revenues and financial solvency. The means for hiding crucial business internals is to employ process views that are subsets of the full in-house conceptual-level processes in the domain of a collaborating party. A process view shields business secrets or irrelevant details and allows an organization to reveal only publicly relevant parts of its private BPs to partner organizations.

Service outsourcing is a business paradigm in which a service-providing organization performs or coordinates parts of a Business Process (BP) of a serviceconsuming organization. These parts were typically performed or coordinated by the service-consuming organization itself. As depicted in Figure 1, the socalled eSourcing-framework $[4,5]$ supports service outsourcing by enabling the flexible construction and structural matching of public, external process views that are extractions of private, internal BPs so that the same activity can occur in different models. A so-called BPaaS-HUB [6] facilitates this matching process during setup time by serving as a repository for the process views and offering automation support during matching, e.g., for background checking potential collaborating counterparties on the fly through mashups.

However, process views and matching relations have been defined considering only the behavior of the process, i.e., the control flow. The human resource perspective involved in the collaboration has hardly been considered in this context, despite its importance for the actual execution of the outsourced activities. Indeed, one of the main reasons of activity outsourcing is the lack of resources with the required skills or the required software within the organization, and the impossibility to acquire them [7].

This position paper works towards that gap by identifying six challenges concerning the research question "how can human resources be managed crossorganizationally assuming a business-process aware collaboration paradigm in VEs?", and two sub-questions deduced from it: (RQ1) How can process views be extended with human resources information? (RQ2) How can the matching of process views with human resources information be automated?

The structure of this paper is as follows. Section 2 describes a scenario that reflects real needs for resource management in eSourcing. Section 3 defines the concepts related to process views and describes the challenges that give answer to 


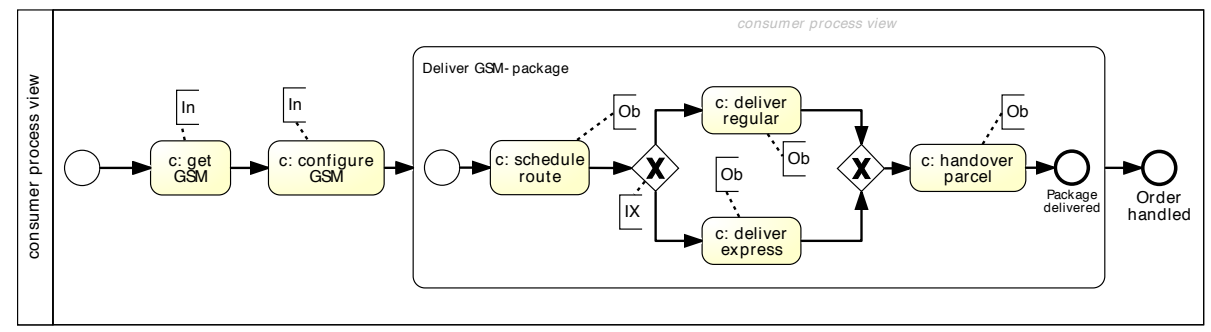

Fig. 2: BPMN process view of consumer. Used annotations: In=invocable, $\mathrm{Ob}=$ observable, $\mathrm{EX}=\mathrm{EXOR}, \mathrm{IX}=\mathrm{IXOR}$. [4].

RQ1. Section 4 follows the same procedure for resource-aware process matching (i.e., RQ2). Finally, Section 5 outlines conclusions and future work.

\section{Motivating Scenario}

In this section, we use concepts related to service outsourcing with process views to describe a motivating scenario that has been adapted from [4]. We also explain the involvement of resources in the process activities and how they are managed. Those details on process views and process-view-based matching that are not relevant for the understanding of the scenario are provided in later sections of the paper.

\subsection{Service Consumer Side}

As part of a larger in-house process located on the internal process layer of a service consumer, Figure 2 shows an example of a telecom sales process, which is a view projection as a service request to the external (public) layer (cf. Figure 1). This projected process view starts with picking a Global System for Mobile (GSM) and configuring it according to customer ${ }^{1}$ demand. In order to speed up the process, the consumer wants that this configuration (activity $c$ : configure $G S M)$ be performed by a person with at least one year of experience configuring GSM devices, and if possible, the same person must be in charge of this activity for all the process executions, i.e., a Binding of Duties (BoD) at process level [8]. Thus, the condition related to experience becomes a necessary (maybe not sufficient) requirement to assign the activity to people at the provider side.

Thereafter, a subprocess is executed to deliver the GSM package, containing the following activities. First, a route is scheduled, and then, either an express or a regular delivery is performed. In order to avoid choosing transportation routes in own favor for reasons external to the enterprise, the person in charge of scheduling the route cannot be same person delivering the package in the

\footnotetext{
${ }^{1}$ The customer is the final beneficiary of the process, hence, in this case the purchaser of the GSM device. The consumer gives service to the customer.
} 
same process execution, i.e., the consumer decides to implement a Segregation of Duties (SoD) policy [8]. The final activity is to hand the GSM package over to the customer, who signs the receipt.

The model representing this process view in Figure 2 uses a specific notation for outsourcing based on the de-facto standard Business Process Model and Notation (BPMN) [9]. We distinguish equally labelled activities that are defined in different domains by prefixing activity labels with a namespace indicator, which is either $c$ : for a consumer, or $p x$ : for a provider. Note that labels may be different but semantically judged as equivalent. If the service provider initiates an activity, it is called observable $[10,11]$ (labeled $O b$ in Figure 2) and if the service consumer initiates an activity, it is called invocable [10,11] (labeled In). We annotate a choice construct to specify whether it is external or internal to a service provider. The annotation $I X$ in Figure 2 denotes that the provider decides on the branch of an exclusive-choice split during enactment, and an $E X$-annotation means the service consumer decides. Notice that information on resources (e.g., assignment conditions, preferences or allocation mechanism) is missing in the model, as resource management has not been considered in process views yet.

All the activities in the process view must be executed by a service provider, from which the set of resources meeting the conditions established are assigned to the activities. From the potential performers assigned to the activities, only some will be selected as actual performers when the process is under execution, considering preferences or further allocation criteria.

However, before starting the collaboration, decisions on who chooses the actual performer for the activities, whether the consumer is notified about it, and who must deal with allocation exceptions, must be negotiated between consumer and provider. In the case at hand, the provider can decide on the final person to be allocated to outsourced activities, as long as she guarantees that all the assignment conditions and the preferences established by the consumer are considered. Nonetheless, the consumer wants to be able to assess performance in the outsourced process considering the people involved so that she can make future decisions. Therefore, the provider is obliged to inform her about the actual performers of the tasks. In case of allocation problems (e.g., illness or holiday season causing a lack of potential performers), the provider is responsible for solving the problem.

\subsection{Service Provider Side}

The model in Figure 3 shows a private process owned by a service provider for matching to the consumer process in Figure 2. The two subprocesses in the BP share some activities and ordering constraints with the consumer process, for example px: get GSM and px: configure GSM. Furthermore, activity labels $c$ : schedule route and $p x$ : determine route can be considered synonyms and, thus, they match. However, the provider may hide internal resource-related information if it is sensitive and should not be seen by the consumer, e.g., specific information related to the organizational structure, which in this case is stored 


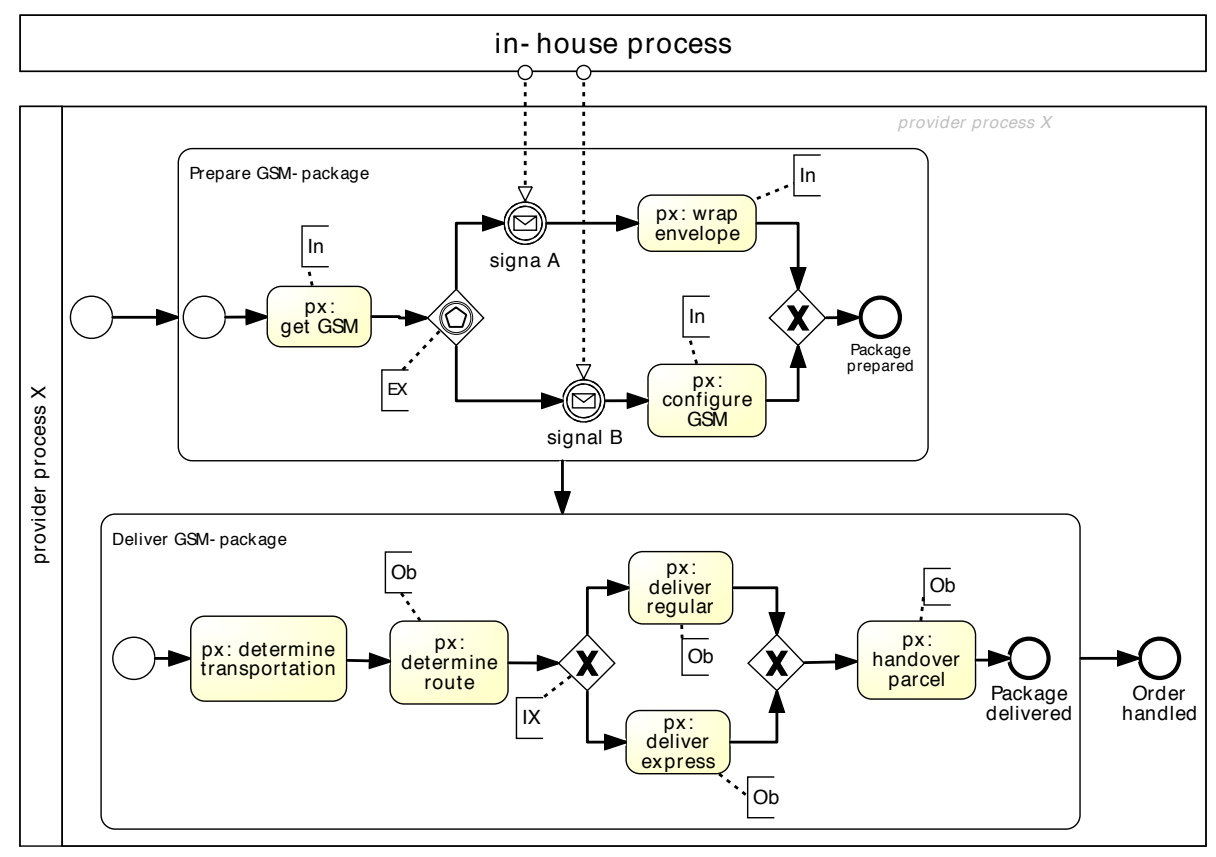

Fig. 3: BPMN process of service provider [4].

in a database and implements a Role-Based Access Control (RBAC) model [12], i.e., a hierarchy of roles. Nevertheless, that information can be internally used to extend the assignment conditions established by the consumer, so that it is opaque to the consumer. For instance, for activity c: configure GSM, the provider may decide that the performer must be someone with role Technician. Therefore, taking into account both consumer's and provider's assignment conditions, all the technicians of the provider with at least one year of experience in GSM configuration are potential performers to execute the activity. Then, the preference specified by the consumer in terms of a $\mathrm{BoD}$ (see above) serves for ranking them, so that the person with highest priority will be allocated to the activity and become its actual performer.

Still, the provider BP contains unobservable and uninvocable activities that are not part of the consumer's process view. In addition, the private BP of the provider in Figure 3 differs from the consumer view in Figure 2 by an eventbased gateway. The two message flows in Figure 3 from the service consumer's in-house domain depict that the service consumer decides the gateway path. The provider process contains additional activities $p x$ : wrap envelope and $p x$ : determine transportation that are opaque for the service consumer during enactment. 


\begin{tabular}{|c|c|c|c|c|c|}
\hline Projection Relation & Omitting & Hiding & Aggregation & $\begin{array}{l}\text { Relation between process view } \\
\text { and internal process }\end{array}$ & Monitoring \\
\hline Black box & & $x$ & $x$ & \begin{tabular}{|l|} 
process view is single \\
observable activity, \\
internal process has \\
no invocable activities \\
\end{tabular} & very limited \\
\hline Glass box & & $x$ & $x$ & \begin{tabular}{|l} 
process view has only multiple \\
observable activities, \\
internal process has \\
no invocable activities \\
\end{tabular} & limited \\
\hline Gray box & $x$ & $\mathbf{x}$ & & \begin{tabular}{|l} 
process view has multiple \\
invocable and observable activities, \\
internal process has invocable \\
and observable activities
\end{tabular} & $\begin{array}{l}\text { limited, } \\
\text { partial, full }\end{array}$ \\
\hline Open box & $x$ & $x$ & $x$ & $\begin{array}{l}\text { process view has multiple invocable } \\
\text { and observable activities, } \\
\text { internal process has invocable } \\
\text { and observable activities } \\
\end{array}$ & $\begin{array}{l}\text { limited, } \\
\text { partial, full }\end{array}$ \\
\hline White box & & & & $\begin{array}{l}\text { process view and internal } \\
\text { process are identical }\end{array}$ & $\begin{array}{l}\text { limited, } \\
\text { partial, full }\end{array}$ \\
\hline
\end{tabular}

Table 1: Projection relations between internal processes and process views [4].

\section{Service Outsourcing with Process Views}

As depicted in Figure 1, the so-called eSourcing framework [5, 4] supports service outsourcing by enabling the flexible construction and structural matching of public, external process views that are extractions of private, internal processes so that the same activity can occur in different models. Concerning the controlflow perspective, these views can be defined in terms of three projection relations.

- Hiding: a set of nodes executed at the internal level are not shown in the process view at the external level.

- Omitting: a set of nodes that do not need to be executed at the internal level are not shown in the process view at the external level, e.g., activities of an EXOR-branch.

- Aggregation: a set of nodes executed at the internal level is shown as a single node in the process view at the external level.

While matching of service offers with service requests requires the process views to be isomorphic, there are projection options termed black-, glass-, gray-, open- and white box [4] between process views and in-house BPs. In Table 1 the extreme projection relations are listed. Black box, glass box, and open box stem from a Web-service outsourcing example by [13] while [14] identifies graybox and white-box projection in a Petri-net study. All other possible projection relations are hybrid forms of these extreme relations.

Black-box projection occurs if the process view contains only a single observable activity that aggregates or hides a set of nodes from the internal process. The internal process does not contain invocable activities as it is not possible to hide or aggregate them. Also EXOR-nodes are no abstraction option as the service consumer cannot monitor or control the internal provider process. 
Glass-box projection occurs if the process view only contains observable activities that hide or aggregate activities from a internal-level process while the process view has no invocable activities. The service consumer merely monitors the internal-process progress.

Gray-box projection occurs if the process view comes into existence through hiding and omitting from the internal proccess. The process view optionally contains both observable and invocable activities while there is no aggregation. The service consumer can monitor the internal process using observable activities in the process view. The service consumer can control the progress of the internal process at th provider side through invocable activities in the process view.

Open-box projection occurs by establishing the process view through hiding, omitting, and aggregation from the internal proccess. The process view contains both observable and invocable activities, allowing a service consumer to monitor and control the progress of the internal process.

Finally, with a white-box projection the process view is identical to the internal process. There is no application of abstraction rules and the service consumer has a direct view on the internal process of the provider including full monitoring and control of the provider-process.

Including human resources in process views such as those mentioned above raises the following challenges:

\section{Challenge 1: Projection relations for human resource assignments}

Description: There might be sensitive information that an enterprise has to hide or omit. For instance, as described in the motivating scenario, the consumer may not be able to show all the organizational structure in order to not violate the Data Protection Act or similar, or she may not want to reveal all the skills of the employees to not take certain risks, e.g., get employees recruited by the provider, or get threatened to post private information. The situation aggravates when a second outsourcing to another supplier is initiated by the provider. Indeed, several situations have occurred in the past related to the publication of sensitive information in outsourcing scenarios. For instance, in 2003 a medical transcriber in Pakistan threatened to post patients' private records online of the California San Francisco Medical Center (UCSM) if certain wages were not paid by an intermediate company that had outsourced to them activities which had been in turn outsourced by the UCSM [15].

Therefore, ensuring privacy control in all the parties involved in an eSourcing collaboration, at the same time as the sharing of information required from each other, is fundamental. Projection rules and relations are defined to guarantee the required degree of privacy for every party involved.

State of the art: In the context at hand, three projection rules and five projection relations are defined to guarantee the required degree of privacy for every party involved [4]. However, only control-flow aspects are considered so far. Studying how to manage resources in the projection rules (paying special attention to aggregation) and in the projection relations, is necessary. New rules and relations may also be identified. As a result, all the parties involved in an 
eSource scenario must be able to hide or omit resource-related information in the projections.

\section{Challenge 2: Expressiveness of the resource assignment language}

Description: A language to define the conditions that the members of an organization must meet to be allowed to participate in an activity, is required in order to specify resource assignments in BPs. Different languages offer different expressiveness regarding the types of conditions that can be defined. The most basic ones are related to organizational entities such as roles, positions, or organizational units; and capabilities associated to people such as their skills or their education. However, advanced features are desirable when dealing with security issues and people experience. In particular, in the domain at hand, the most interesting conditions that should be covered by the assignment language can be seen as five different sub-challenges related to expressiveness, namely:

Challenge 2.1: Capability-based resource assignment The ability to define assignment conditions based on the capabilities associated to resources, e.g., their skills or their education.

Challenge 2.2: Security-aware resource assignment The ability to define access-control constraints to configure security in the execution of outsourced process activities, e.g., SoD and BoD [8].

Challenge 2.3: History-aware resource assignment The ability to define the assignment of resources based on historical information on past process executions. This is especially relevant in long-term outsourcing scenarios where the consumer may want to assign resources depending on their past performance in similar activities.

Challenge 2.4: Preferences in resource assignment The ability to define preferences on the people that can execute an outsourced activity to give priority to the most suitable person, so that the provider must try to allocate the work to the first person in the priority ranking generated according to the organizational model of the company and the characteristics of its members. Preferences have implications in order challenges (see Challenge 3).

Challenge 2.5: Task duties in resource assignment The ability to define different degrees of responsibility associated to an activity, e.g., a person responsible for its execution, a person accountable for it, or a person providing external information required for its completion. 
Note that the assignment specified by the consumer defines the minimum set of conditions that must be fulfilled. Further conditions can be added by the provided internally, of which the consumer might not be aware (cf. Section 2).

State of the art: The languages for the definition of resource assignments typically support only a subset of the features mentioned above. For instance, BPMN [9] and WS-Human Task [16]/BPEL4People [17] do not allow defining accesscontrol constraints natively. In the case of BPMN, this could be done by using another language with the notation, as proposed in [18]. WS-Human Task and BPEL4People allow defining different task duties for the BP activities, which constitute a subset of the task duties defined in so-called RASCI matrices [19]. Yet Another Workflow Language (YAWL) [20] and Architecture of Integrated Information Systems (ARIS) [21] do not take into account information about past executions or the existence of different task duties associated to an activity. Finally, some approaches $[8,22]$ deal with security and history-based aspects but they do not support the definition of conditions about resource capabilities because they are based on the RBAC model [12], which relies only on organizational roles. Resource Assignment Language (RAL) [18, 23] could be a candidate to achieve this challenge, as it provides support for features 2.1, 2.2, 2.3 and 2.5, as well as conditions based on organizational entities.

Regarding preferences, different types of preferences and different formalisms to define them have been used in different domains [24]. For example, quantitative preferences have been discussed in economics, operations and web systems [25-28], while qualitative preferences have been the focus of artificial intelligence and database research [29-31]. Some approaches for the discovery and ranking of semantic web services cover both types of preferences. Specifically, Semantic Ontology of User Preferences (SOUP) [32] has been used to define preferences in Business Process Management (BPM) together with RAL [33], thus bridging and existing gap in intra-organizational process-aware resource management.

\section{Challenge 3: Control over the allocation of outsourced activities}

Description: The consumer is typically in charge of defining the minimum set of resource assignment conditions for the outsourced activities, but an agreement on who decides on the allocation and the information publicly available for the consumer is also necessary. There are at least three options: (i) the consumer does not know who in the provider performs the work; (ii) the consumer cannot make a decision on the worker for the outsourced activities, but he gets informed about who performed the task after completion; (iii) the consumer decides who must execute the activity from the set of potential performers of the provider, thus being totally aware of the allocation procedure.

This is specially relevant when security policies must be implemented in the process (e.g., the Dynamic SoD (DSoD) and Static BoD (SBoD) constraints applied in the motivating scenario), as it is necessary to take into account who has participated in previous executions of the process activities. The degree of 


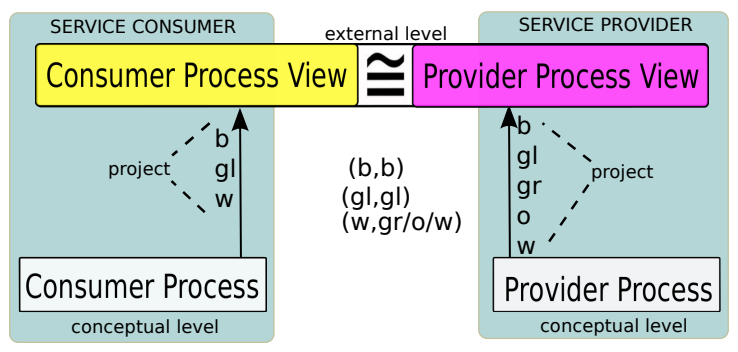

Fig. 4: Valid collaboration configurations [4].

transparency in resource allocation must, thus, be agreed upon between consumer and provider, as privacy concerns also play a role in this decision (see Challenge 1).

Also related to this challenge is the question of who must handle exceptions related to resource allocation in case of a lack of potential performers available. Under those circumstances, the consumer might want to modify the resource assignments of the outsourced activities that are affected, or the resolution of the conflict might fall on the provider, which can, e.g., implement some substitution policy always guaranteeing that the assignment conditions remain fulfilled. In the worst case, a second outsourcing might be required.

State of the art: In [4], the concepts of invocable and observable activities in eSourcing scenarios were introduced related to the party involved in the collaboration that must initiate the work in the outsourced activities. However, there was no information about who selects the specific person that works in the activity and the related information that is made visible to the other party. Similarly, exception handling is disregarded and becomes especially relevant when resources are taken into account. Therefore, the extension of these concepts to consider resource allocation and reaction to potential exceptions is an open issue.

\section{Matching between Process Views}

The service consumer on the left and service provider on the right of Figure 4 associate their process views with one another using the matching relations of Section 3 relating their process views to respective underlying internal processes. The figure shows only certain combinations of projection (cf. Section 3) and matching relations that are possible for process views at the external level. The internal levels show the useful projection relations near the projection arrow for the service consumer and provider. In the centre, the tuples with projection combinations are permitted combinations for matching process views on the external level. If the service consumer performs a black-box projection, the provider must also use black-box projection. All other projections do not yield a single aggregation externally.

If the service consumer uses a glass-box projection, it imposes a limitation on the service provider that he can only use observable activities. Thus, the latter 
must also respond with a glass-box projection. However, when a service consumer uses open-, or white-box projection, the service provider has the options of a gray-box, open-box, or white-box projection. The contained invocable activities eliminate black-box and glass-box projections for the service provider.

A service consumer cannot use a gray-box or open-box projection as the process view may omit an invocable node from the internal process. However, that creates a problem during enactment time when the consumer invokes a node from her internal process level without the event being able to bridge into the domain of the service provider because the required externally projected equivalent invoke node is missing in the process view.

The previous matching relations have been defined disregarding the existence of resource assignments and, thus, the need of managing human resources. With resource-aware process views, the matching mechanisms between the process views of consumer and provider should be extended in several ways. In particular, the following challenges have been identified:

\section{Challenge 4: Consideration of the resource perspective in the matching relations of the process views}

Description: As consumer and provider develop process views independently, these are not necessarily in line. However, they must be compatible and must meet the degree of matching needed for the communication, which varies in different scenarios, e.g., if an enterprise outsources part of its BP to a supplier, synchronization might not be required, but the monitoring of the progress at the provider side might be desired [4]. Therefore, the resource perspective must be taken into account also when defining matching relations, which may change with respect to those defined considering only the control flow of the process. For instance, the different degrees of matching that can be found between the resource assignments defined by consumer and provider has to be studied.

State of the art: As described above, Eshuis et al. identified three matching relations related to the BP control flow [4], but resource assignments were disregarded. These matching relations should be re-studied and adapted considering resource-related information. New matching relations stemming from the consideration of resource assignments in the process activities might be identified.

\section{Challenge 5: Different vocabulary in the parties involved}

Description: Natural language is very rich and the terminology used to refer to concepts vary depending on the country, the region and even, the person. Searching for term matching in different languages may also be challenging. These issues affect organizational metamodels too, in which the same concept is sometimes referred to in two different ways (synonyms) and two terms are usually used to mean the same concept (homonyms). Capabilities and/or qualifications or professional regulations tend to receive different names in different contexts or domains as well. All this might incur in a lack of understanding between the parties involved in a collaboration. Therefore, ensuring that consumer 
and provider use the same vocabulary (or at least a vocabulary that both can interpret in the same way) is crucial for eSourcing success.

State of the art: A domain-specific dictionary or thesaurus upon which all the parties must agree, can be used to provide the terms with a specific meaning. Several approaches can be used to build it, e.g. Levenshtein distance [34] or Lin metric [35], which provide meaningful semantics of the concepts and their equivalence. There are dictionaries such as WordNet [36] and BabelNet [37], on which we can rely. Indeed, these tools have been used in previous approaches to automatically generate BP models from textual descriptions [38], and vice versa [39], producing good results. For the purpose at hand, Natural Language Processing (NLP) techniques must be put in place.

\section{Challenge 6: Automation in the matching of process views}

Description: Algorithms to automate the matching check between the process views of the parties involved in a collaboration are required. This would allow ensuring not only that process views are compatible with each other before initiating a collaboration, but also that the checking can be repeated during process execution if something changes in one of the parties, at a lower time than if having to perform it manually. Therefore, this challenge contributes to save time and the subsequent human cost, at the same time as it helps to improve the quality of the service and provides flexibility to perform changes whose consequences can be straightforwardly checked. Please, notice that Challenge 5 must also be considered to guarantee that process matching meets the requirements.

State of the art: General concepts from ontology matching [40] have recently been adopted for the automatic matching of processes [41]. The challenge at hand is in this context that activities can be labeled with verb phrases in heterogeneous ways [42], which goes beyond what ontology matching can readily handle. The control flow perspective provides the opportunity to constrain the search space for matches [43]. Improvement directions have been investigated in [44, 45, 6]; however, without taking the resource perspective explicitly into account.

\section{Conclusions}

In this paper, we have explored how human resources fit in a process-aware crossorganizational setting in order to answer the question "how to manage human resources cross-organizationally assuming a business-process aware collaboration paradigm in VEs?". The starting point is a description of service outsourcing based on the definition of process views and different matching relations to check process compatibility of the parties involved, as introduced in the eSourcing framework $[5,4]$.

In particular, we have described a scenario based on a real case in which the resource perspective is reflected. Then, we have identified a set of challenges related to the two main elements involved in eSourcing scenarios, specifically three challenges related to the integration of human resources information in process 
views, and three challenges associated to the automatic matching between process views with resources information. The state of the art of related work has been summarized for every challenge.

Altogether, the problem is complex because many issues need to be addressed (e.g., a resource assignment language, a dictionary and a method for the disambiguation of natural language), and sensitive aspects such as privacy and security must be dealt with. However, we have found approaches for intra-organizational human resource management that provide (partial) support for (some of) the desired features. For instance, RAL [18] could potentially be used to specify expressive resource assignments, and matching algorithms developed to compare BP models similarity [41] could be adapted to the eSourcing domain.

Nonetheless, in this paper we have assumed that a process activity is always executed by a single person, i.e., individual allocation. In reality, many activities require collaborative work, especially in domains such as software development. Therefore, team work should also be studied in this context.

\section{References}

1. N. Mehandjiev and P. Grefen, eds., Dynamic Business Process Formation for Instant Virtual Enterprises. Springer, 2010.

2. F. D. P. E. Pimentel and G. Zavattaro, "Service-oriented and cloud computing," 2012.

3. S.-W. Chou and C.-H. Chiang, "Understanding the formation of software-as-aservice (saas) satisfaction from the perspective of service quality," Decision Support Systems, 2013.

4. R. Eshuis, A. Norta, O. Kopp, and E. Pitkänen, "Service Outsourcing with Process Views," IEEE Transactions on Services Computing, vol. 99, 2013.

5. A. Norta and R. Eshuis, "Specification and verification of harmonized businessprocess collaborations," Information Systems Frontiers, vol. 12, pp. 457-479, 2010.

6. A. Norta and L. Kutvonen, "A Cloud HUB for Brokering Business Processes as a Service: A Rendezvous Platform that Supports Semi-automated Background Checked Partner Discovery for Cross-Enterprise Collaboration," in SRII Global Conference (SRII), 2012 Annual, pp. 293-302, 2012.

7. D. Straub, P. Weill, and K. S. Schwaig, "Strategic Dependence on the IT Resource and Outsourcing: A Test of the Strategic Control Model," Information Systems Frontiers, vol. 10, pp. 195-210, Apr. 2008.

8. M. Strembeck and J. Mendling, "Modeling process-related RBAC models with extended UML activity models," Inf. Softw. Technol., vol. 53, pp. 456-483, May 2011.

9. OMG, "BPMN 2.0," recommendation, OMG, 2011.

10. J. Ebert and G. Engels, "Observable or invocable behaviour - you have to choose!," Technical report 94-38, Leiden University, 1994.

11. G. Preuner and M. Schrefl, "Requester-centered composition of business processes from internal and external services," Data Knowl. Eng, vol. 52, no. 1, pp. 121-155, 2005.

12. I. American National Standards Institute, "Role-Based Access Control. ANSI INCITS 359-2004." http://csrc.nist.gov/rbac, February 2004. 
13. P. Grefen, H. Ludwig, A. Dan, and S. Angelov, "An analysis of web services support for dynamic business process outsourcing," Information and Software Technology, vol. 48, no. 11, pp. 1115-1134, 2006.

14. A. Norta, Exploring Dynamic Inter-Organizational Business Process Collaboration. PhD thesis, Technology University Eindhoven, Department of Information Systems, 2007.

15. M. Brown, "Privacy and Outsourcing: Evolving Concerns." Newsletter, May 2007.

16. "Web Services-Human Task (WS-HumanTask) v1.1," tech. rep., OASIS, 2010.

17. "WS-BPEL Extension for People (BPEL4People)," tech. rep., OASIS, 2009.

18. C. Cabanillas, M. Resinas, and A. Ruiz-Cortés, "RAL: A High-Level User-Oriented Resource Assignment Language for Business Processes," in Business Process Management Workshops (BPD'11) (F. Daniel, K. Barkaoui, and S. Dustdar, eds.), pp. 50-61, Springer, 2011.

19. Website, "The RASCI matrix." http://www.ha-ring.nl/en/doc_en/ rasci-matrix, Last accessed in January 2014.

20. W. M. P. van der Aalst and A. H. M. ter Hofstede, "YAWL: Yet Another Workflow Language," Inf. Syst., vol. 30, no. 4, pp. 245-275, 2005.

21. ARIS, "RACI, ARIS Community Website." http://www.ariscommunity.com/ raci, 2012.

22. F. Casati, S. Castano, and M. Fugini, "Managing Workflow Authorization Constraints through Active Database Technology," Information Systems Frontiers, vol. 3, pp. 319-338, September 2001.

23. C. Cabanillas, M. Resinas, and A. Ruiz-Cortés, "Designing Business Processes with History-Aware Resource Assignments," in BPM 2012 Workshops (BPD'12) (M. L. Rosa and P. Soffer, eds.), vol. 132, pp. 101-112, Springer, 2012.

24. C. Domshlak, E. Hüllermeier, S. Kaci, and H. Prade, "Preferences in AI: An overview," Artif. Intell., vol. 175, no. 7-8, pp. 1037-1052, 2011.

25. P. C. Fishburn, Utility theory for decision making. Wiley, 1970.

26. R. L. Keeney and H. Raiffa, Decisions with multiple objectives: Preferences and value tradeoffs. Cambridge Univ Press, 1993.

27. R. Agrawal and E. L. Wimmers, "A Framework for Expressing and Combining Preferences," in SIGMOD Conference, pp. 297-306, ACM, 2000.

28. L. Zeng, B. Benatallah, A. H. H. Ngu, M. Dumas, J. Kalagnanam, and H. Chang, "QoS-Aware Middleware for Web Services Composition," IEEE Trans. Software Eng., vol. 30, no. 5, pp. 311-327, 2004.

29. C. Boutilier, R. I. Brafman, C. Domshlak, H. H. Hoos, and D. Poole, "CP-nets: A Tool for Representing and Reasoning with Conditional Ceteris Paribus Preference Statements," J. Artif. Intell. Res. (JAIR), vol. 21, pp. 135-191, 2004.

30. W. Kießling, "Foundations of Preferences in Database Systems," in VLDB, pp. 311-322, VLDB Endowment, 2002.

31. J. Chomicki, "Preference formulas in relational queries," ACM Trans. Database Syst., vol. 28, no. 4, pp. 427-466, 2003.

32. J. M. García, D. Ruiz, and A. Ruiz-Cortés, "A Model of User Preferences for Semantic Services Discovery and Ranking," in $E S W C$ (2) (L. Aroyo, G. Antoniou, E. Hyvnen, A. Teije, H. Stuckenschmidt, L. Cabral, and T. Tudorache, eds.), vol. 6089 of LNCS, pp. 1-14, Springer Berlin Heidelberg, 2010.

33. C. Cabanillas, J. M. García, M. Resinas, D. Ruiz, J. Mendling, and A. Ruiz-Cortés, "Priority-Based Human Resource Allocation in Business Processes," in ICSOC 2013 (S. Basu, C. Pautasso, L. Zhang, and X. Fu, eds.), vol. 8274, pp. 374-388, Springer, 2013. 
34. S. Sahinalp, M. Tasan, J. Macker, and Z. Ozsoyoglu, "Distance based indexing for string proximity search," in Data Engineering, 2003. Proceedings. 19th International Conference on, pp. 125-136, March 2003.

35. D. Lin, "An Information-Theoretic Definition of Similarity," in International Conference on Machine Learning, ICML '98, pp. 296-304, Morgan Kaufmann Publishers Inc., 1998.

36. C. Fellbaum, WordNet. Blackwell Publishing Ltd, 2012.

37. R. Navigli and S. P. Ponzetto, "BabelNet: Building a Very Large Multilingual Semantic Network," in Annual Meeting of the Association for Computational Linguistics, ACL '10, pp. 216-225, 2010.

38. F. Friedrich, J. Mendling, and F. Puhlmann, "Process Model Generation from Natural Language Text," in International Conference on Advanced Information Systems Engineering (CAiSE) (H. Mouratidis and C. Rolland, eds.), Lecture Notes in Computer Science, pp. 482-496, Springer Berlin Heidelberg, 2011.

39. H. Leopold, J. Mendling, H. A. Reijers, and M. L. Rosa, "Simplifying process model abstraction: Techniques for generating model names," Inf. Syst., vol. 39, pp. 134-151, 2014

40. J. Euzenat and P. Shvaiko, Ontology matching. Springer, 2007.

41. M. Weidlich, R. Dijkman, and J. Mendling, "The ICoP Framework: Identification of Correspondences Between Process Models," in International Conference on Advanced Information Systems Engineering (CAiSE) (B. Pernici, ed.), CAiSE'10, pp. 483-498, Springer-Verlag, 2010.

42. H. Leopold, S. Smirnov, and J. Mendling, "On the refactoring of activity labels in business process models," Inf. Syst., vol. 37, no. 5, pp. 443-459, 2012.

43. H. Leopold, M. Niepert, M. Weidlich, J. Mendling, R. Dijkman, and H. Stuckenschmidt, "Probabilistic Optimization of Semantic Process Model Matching," in $B P M$ (A. P. Barros, A. Gal, and E. Kindler, eds.), BPM'12, pp. 319-334, Springer, 2012.

44. C. Klinkmueller, I. Weber, J. Mendling, H. Leopold, and A. Ludwig, "Increasing Recall of Process Model Matching by Improved Activity Label Matching," in BPM (F. Daniel, J. Wang, and B. Weber, eds.), Lecture Notes in Computer Science, pp. 211-218, Springer Berlin Heidelberg, 2013.

45. M. Weidlich, T. Sagi, H. Leopold, A. Gal, and J. Mendling, "Predicting the Quality of Process Model Matching," in BPM (F. Daniel, J. Wang, and B. Weber, eds.), pp. 203-210, Springer, 2013. 